\title{
Síndrome de Burnout em profissionais de enfermagem em um pronto-socorro do Distrito Federal (DF)*
}

\section{Burnout Syndrome in professional nursing in a first aid the Federal District (DF)}

Thamires Ita de Lucena' Linconl Agudo Oliveira Benito ${ }^{2}$
* Recebido em: 01/10/2014. Aprovado em: 25/05/2015.

1 Acadêmica em Enfermagem do Centro Universitário de Brasília - UniCEUB.

2 Mestre em Gerontologia pela Universidade Católica de Brasília (UCB). Docente do UniCEUB.

\section{Resumo}

A enfermagem é classificada como a quarta ocupação que tem maior taxa de esgotamento no domínio público. A Síndrome de Burnout (SB) relacionada ao cansaço do trabalhador é determinada como síndrome psicológica em consequência da inquietação emocional duradoura no trabalho, constituindo-se de três aspectos: Exaustão Emocional (EE), Despersonalização (DE) e Realização Profissional (RP). O principal objetivo deste artigo consiste em identificar sua ocorrência entre a enfermagem na assistência ao pronto-socorro. Trata-se de estudo transversal, com abordagem quantitativa, realizando aplicação de questionários para o levantamento de variáveis sócio-demográficas e o Inventário de Maslach Burnout (MBI). A amostra constitui-se de 69 profissionais de enfermagem, sendo a maioria do sexo feminino, os resultados apresentaram níveis mais elevados de $\mathrm{EE}$ e $\mathrm{DE}$ quando comparados à RP. O estudo demonstrou pontos cruciais nos quais se deve optar pela melhora do trabalho da equipe de enfermagem.

Palavras-chave: Enfermagem. Profissionais. Síndrome de Burnout.

\begin{abstract}
Nursing is ranked as the fourth occupation that has higher depletion rate in the public domain. The Burnout Syndrome (SB) is related to fatigue of the worker is determined as a psychological syndrome as a result of enduring emotional upheaval at work, constituting three aspects: Emotional Exhaustion (EE), depersonalization (DE) and Professional Achievement (RP). The main objective is to identify factors among nursing care in the emergency room. This cross-sectional study with a quantitative approach, conducting questionnaires for the collection of socio-demographic variables and the Maslach Burnout Inventory (MBI). The sample consisted of 69 nurses, mostly female, the results showed higher levels of EE and DE compared to RP. The study demonstrated the crucial points where you should opt for the improvement of the work of the nursing team.
\end{abstract}

Keywords: Nursing. Professional. Burnout Syndrome. 


\section{Introdução}

Na evolução do mundo moderno, o trabalho humano possui indiscutível importância tanto para o desenvolvimento social como também para o econômico de uma sociedade organizada. Este se constitui essencial para a existência e subsistência, do indivíduo, que, por meio de suas atividades, alcança efeitos positivos e produtivos de suas capacidades e potencialidades, além de dedicação e empenho (RODRIGUES et al., 2012).

O exercício profissional enquanto intermédio de formação coletiva, tendo sua importância tanto financeira quanto cultural, mérito crucial na forma de vida das pessoas, e em sua saúde física e mental. Portanto, comprova-se que os trabalhadores em todas as dimensões e categorias necessitam conhecer melhor, para prevenir as várias formas de mecanismos patológicos que estão vulneráveis, no desenvolvimento de suas práticas laborativas (RODRIGUES et al., 2012).

O esgotamento no serviço é resultante da anexação da pessoa nessa situação, pois, com o tempo, o desenvolvimento do trabalho, além de proporcionar mudança, condecoração e autonomia, por outro lado, pode, se não for criteriosamente cuidado e protegido, originar contratempos como, por exemplo, desgosto, desapego, insensibilidade e estresse. Nesse sentido, o trabalho deve ser agradável e ter condições motivacionais para o ser humano (BATISTA; BIANCHI, 2006).

$\mathrm{Na}$ área da saúde, suas profissões componentes registram o terceiro lugar enquanto vencedores na constituição e formação de estresse. Nessa circunstância, a categoria profissional de enfermagem é classificada enquanto a quarta ocupação que apresenta maior taxa de esgotamento no domínio público. Desde a sua origem e contemporaneamente, o exercício dos trabalhadores em saúde se representa e se apresenta intimamente conectado ao sofrimento, a patologias e também ao óbito (FARIAS et al., 2011).

A expressão "burnout" foi empregada inicialmente no ano de 1974, referida pelo psicólogo Herbert J. Freudenberger, relatando situação observada em jovens empregados de uma clínica na cidade de Nova York, Estados Unidos, no que se refere a substâncias químicas. Dessa forma, a síndrome de Burnout (SB) se encontra intimamente relacionada aos profissionais de saúde em flexíveis, com tratamento distante e insensível para com os pacientes (MOREIRA et al., 2009).

A SB se caracteriza enquanto fenômeno composto por três (03) dimensões constitutivas, sendo elas, a exaustão emocional (ou seja, o fator central do esgotamento, que se caracteriza pelo sentimento de desgaste emocional e também pela falta de energia), no segundo momento a despersonalização (relacionada à insensibilidade emocional observada enquanto estratégia defensiva e que também se dá quando o profissional em questão passa a tratar os seus clientes e também colegas como objetos) ainda e a reduzida ou falta de realização pessoal (sentimento de incompetência e de inadequação). (MASLACH; JACKSON, 1981).

O esgotamento nem sempre é negativo, porém o esgotamento distendido consiste em um das consequências do estresse, que pode ocasionar a SB. Nesse sentido, o estresse pode ou não acarretar consumo das energias do corpo humano no geral, resultando da proporção, da extensão e da fragilidade do ser humano, tendo sua competência em gerenciá-lo (FRANÇA, 2010).

A SB se encontra relacionada ao cansaço do trabalhador, sendo determinada enquanto síndrome psicológica, em consequência da inquietação emocional duradoura no trabalho, constituindo-se de três (03) aspectos, a Exaustão Emocional (EE), referindo a um esgotamento de energia, a Despersonalização (DE) relacionada à insensibilidade com os clientes e colegas de trabalho e a Realização Profissional (RP) associado à incapacidade no desenvolvimento profissional (TAMAYO, 2009).

Nessa perspectiva, o presente trabalho busca identificar a ocorrência da SB entre os profissionais da área de enfermagem na assistência ao pronto-socorro (PS).

\section{Metodologia}

Tratou-se de estudo classificado enquanto transversal e descritivo, contemplando abordagem quantitativa, sendo realizado entre o mês de março a abril do ano de 2014. A amostra pesquisada foi composta por um universo de 69 profissionais de enfermagem, sendo estes da categoria de enfermeiros e técnicos em enfermagem da Secretaria de Estado de Saúde do Distrito Federal (SES-DF) e, lotados em um pronto-socorro (PS) com sede na cidade satélite de Planaltina, Distrito Federal (DF), e que atuam na assistência de enfermagem no turno matutino, vespertino e noturno. 
Observaram-se os seguintes critérios de inclusão dos sujeitos para este estudo: colaboradores voluntários,, pertencentes ao sexo feminino ou masculino, profissionais da área de enfermagem no setor do PS. Para a participação na pesquisa foi solicitado que os interessados assinassem livremente o Termo de Consentimento Livre e Esclarecido (TCLE). Os dados foram coletados por meio da aplicação de dois (02) instrumentos de coleta de dados (ICD). O primeiro observou as características socioeconômicas, demográficas e trabalhistas dos profissionais, tais como: faixa etária, escolaridade, renda e tempo de serviço, e o segundo procurou mensurar a existência da SB entre os profissionais, utilizando para essa atividade o Inventário de Maslach Burnout (MBI).

Nesse sentido, o referido ICD se constitui enquanto o instrumento mais utilizado para avaliar a SB, independentemente das características ocupacionais da amostra e de sua origem, foi elaborado pela Dra. Christina Maslach e a Dra. Susan Jackson em 1978. O ICD foi adaptado por Tamayo em 1978 para a Língua Portuguesa. Sua construção partiu de duas (02) dimensões, ou seja, a exaustão emocional e a despersonalização, e a terceira $\left(3^{a}\right)$ dimensão, a realização profissional, surgiu após estudo desenvolvido com centenas de pessoas de uma ampla gama de profissionais.

O MBI é autoinforme, pois, para ser respondido por uma escala de frequência de cinco (05) pontos, que vai de um (nunca) até cinco (sempre). O MBI é composto por 22 itens que avaliam as três (03) dimensões independentes entre si que, sendo elas, a EE, a DE e a RP. Desses 22 itens, nove (09) são em relação à EE, cinco (05) são em relação à DE e oito (08) são em relação à RP.

Com base no questionário aplicado, o cálculo dos escores ocorre de acordo com o seguinte método. A pontuação em cada subescala foi obtida por meio da soma dos valores dos respectivos. Para isso, considerou-se que, na subescala de EE pontuação igual ou maior que vinte e seis (26), sendo indicativo de alto nível de exaustão, o intervalo compreendido entre 16-25 corresponde a valores moderados, e valores iguais ou menores que 15 indicam baixo nível (FRANÇA, 2010).

$\mathrm{Na}$ subescala de $\mathrm{DE}$, a pontuação igual ou superior a 13 se constitui enquanto sendo nível alto, a pontuação entre 07-12 representa nível moderado e a pontuação igual ou menor que 06 aponta baixo grau de despersonalização. A subescala de RP apresenta medida inversa, ou seja, pontuações iguais ou maiores que 31 , o que indica alto sentimento de realização profissional e consequentemente, baixo nível de esgotamento. Pontuações entre 32-36 indicam moderado nível de realização e somatório de 39 acima, indicam baixo nível de desgaste (FRANÇA, 2010).

No agrupamento efetuado por Maslach e Jackson (1978), os procedimentos para obtenção de resultados foram efetuados a priori, a obtenção de altas pontuações em EE e DE e baixa pontuação em RP sugerem a presença de esgotamento profissional em alto grau (FRANÇA, 2010).

O estudo realizou a análise de dados por meio da análise estatística exploratória e descritiva e com o cálculo dos respectivos percentuais. Foram confeccionadas três (03) tabelas, organizadas e processadas eletronicamente pelo programa Microsoft Excel 2007 ${ }^{\circledR}$, pertencente ao pacote Microsoft Office $2007^{\circledR}$ for Windows ${ }^{\circledast}$ Para a execução das análises estatísticas, foi utilizado o programa SPSS ${ }^{\circledR}$ (Statistical Package for the Social Sciences ${ }^{\circledast}, \mathrm{IBM}^{\circledast}$ ) em sua versão de número 20.0 for Windows ${ }^{\oplus}$, licenciado para desenvolvimento de projetos acadêmicos.

Os participantes assinaram o Termo de Consentimento Livre e Esclarecido (TCLE) e a pesquisa acompanhou e respeitou integralmente os preceitos constantes junto a Resolução n. 466/2012 da Comissão Nacional de Ética em Pesquisa (CONEP), obtendo aprovação do Comitê de Ética em Pesquisa (CEP) da Fundação de Ensino e Pesquisa em Ciências da Saúde (FEPECS), do Governo do Distrito Federal (GDF), com número de registro CAAE: 25905014.6.0000.5553.

\section{Resultados}

Durante o processo da coleta de dados e, em um universo de 129 profissionais de enfermagem, 53,50\% $(\mathrm{n}=69)$ responderam aos questionamentos propostos, $15,50 \%(n=20)$ se encontravam de férias, $19,40 \%(n=25)$ foram extraviados e 11,60 $(n=15)$ se recusaram a responder ao ICD, conforme exposto junto à tabela 1 .

Nesse sentido, participaram do presente estudo um quantitativo de $11,60 \%(n=08)$ constituído por profissionais enfermeiros e $88,40 \%(n=61)$ de técnicos em enfermagem, regularmente registrados enquanto funcionários da unidade de PS avaliado. 
Tabela 1 - Distribuição das variáveis com relação à pesquisa aplicada aos profissionais de enfermagem de uma unidade de Pronto Socorro (PS). Planaltina. Brasília (D.F.), 2014. $(n=129)$ :

\begin{tabular}{lcc}
\hline Situação & Frequência & $\%$ \\
\hline Universo de atores sociais & 129 & 100,00 \\
Participantes da pesquisa & 69 & 53,50 \\
Ausentes na pesquisa & 20 & 15,50 \\
Extraviados & 25 & 19,40 \\
Recusados & 15 & 11,60 \\
\hline
\end{tabular}

Fonte: Do Autor.

Tabela 2 - Perfil socioeconômico dos sujeitos da pesquisa. Planaltina. Brasília (D.F.), 2014. (N=69).

\begin{tabular}{lll}
\hline Sexo & $\mathbf{N}$ & $\%$ \\
\hline Feminino & 43 & 62,3 \\
Masculino & 26 & 37,7 \\
\hline Faixa etária & & \\
\hline $20-40$ & 53 & 76,80 \\
$41-60$ & 16 & 23,20 \\
\hline
\end{tabular}

Profissional

Técnico de Enfermagem $61 \quad 88,40$

Enfermeiro

$08 \quad 11,60$

\begin{tabular}{|c|c|c|}
\hline Estado Civil & & \\
\hline Casado & 38 & 55,1 \\
\hline Solteiro & 20 & 29,0 \\
\hline União Estável & 10 & 14,5 \\
\hline Divorciado & 01 & 1,4 \\
\hline \multicolumn{3}{|l|}{ Número de filhos } \\
\hline 0 filho & 15 & 21,7 \\
\hline 1-2 filhos & 42 & 60,8 \\
\hline $3-4$ filhos & 12 & 17,8 \\
\hline \multicolumn{3}{|l|}{ Renda Mensal * } \\
\hline 2 até 4 & 21 & 30,4 \\
\hline 5 até 7 & 34 & 49,2 \\
\hline Até 10 & 14 & 20,3 \\
\hline \multicolumn{3}{|l|}{ Escolaridade } \\
\hline Ensino Médio Completo & 29 & 42,0 \\
\hline Ensino Superior Incompleto & 12 & 17,4 \\
\hline Ensino Superior Completo & 22 & 31,9 \\
\hline Superior Latu-sensu & 06 & 8,7 \\
\hline \multicolumn{3}{|l|}{ Jornada de Trabalho } \\
\hline Plantonista & 69 & 100 \\
\hline \multicolumn{3}{|l|}{ Carga Horária } \\
\hline 20 horas & 15 & 21,7 \\
\hline 40 horas & 49 & 71,0 \\
\hline Outras & 05 & 7,2 \\
\hline
\end{tabular}

\section{Tempo de serviço}

$<1$ ano - 4 ano

5 anos -9 anos

$>10$ anos

39

21

09

\section{Outro emprego}

\begin{tabular}{lll}
\hline Não & 59 & 85,5
\end{tabular}

Sim

10

14,5

Tempo de Formação

$<1$ ano -5 anos

6 anos -15 anos

16 anos -25 anos

22

31,7

53,5

37

14,4
Em relação ao sexo dos atores sociais participantes do presente estudo, a maior frequência do universo analisado se constituiu de profissionais de enfermagem do sexo feminino, que registraram um total de $62,30 \%$ $(n=43)$, com predomínio de faixa etária entre 20 e 40 anos, que equivale a $76,80 \%(\mathrm{n}=53)$. A maioria dos profissionais analisados referiu ser casada, efetivando um quantitativo de $55,10 \%(\mathrm{n}=38)$, e possuir renda mensal entre 05 e 07 salários mínimos, somando um total de 49,20\% ( $n=34)$, conforme exposto junto à Tabela 2.

$\mathrm{Na}$ categoria que analisou a escolaridade dos sujeitos da pesquisa, foi evidenciado predomínio daqueles que declararam possuir ensino médio completo (EMC), registrando um total de $42 \%(\mathrm{n}=29)$. Já em relação ao tempo de formação, a maior frequência registrada foi aquela compreendida entre 06 e 15 anos, constituindo $53,50 \%(n=37)$.

Em relação à categoria que analisou o tempo de serviço na instituição participante do estudo, foi encontrada a maior frequência no período compreendido ao espaço inferior a um (01) e quatro (04) anos, correspondendo a $56,50 \%(n=39)$. Dos profissionais entrevistados $71 \%$ $(\mathrm{n}=49)$ cumpriam 40 horas semanais, e $85,50 \%(\mathrm{n}=59)$ declararam não possuir outro vínculo empregatício.

Conforme exposto junto à tabela 3 , foi demonstrada a distribuição por níveis das dimensões propostas para o diagnóstico da $\mathrm{SB}$, que resultaram em EE apresentando maior frequência no nível moderado, registrando $65,20 \%$ $(\mathrm{n}=45)$, na segunda ( $\left.2^{\mathrm{a}}\right)$ colocação foi encontrado o nível alto com $21,75 \%(\mathrm{n}=15)$ e o nível baixo somando um total de $13,05 \%$ (n=09).

Tabela 3 - Distribuição das três (03) dimensões e níveis para o diagnóstico da Síndrome de Burnout, em relação aos profissionais de Enfermagem de um Pronto Socorro. Planaltina. Distrito Federal (D.F.). 2014. ( $\mathrm{N}=69)$.

\begin{tabular}{|c|c|c|c|c|c|c|}
\hline \multirow{2}{*}{$\begin{array}{l}\text { Níveis - Síndrome } \\
\text { de Burnout }\end{array}$} & \multicolumn{2}{|c|}{$\mathrm{EE}^{*}$} & \multicolumn{2}{|c|}{$D E^{* *}$} & \multicolumn{2}{|c|}{$R P^{* * *}$} \\
\hline & $\mathrm{N}$ & $\%$ & N & $\%$ & $\mathrm{~N}$ & $\%$ \\
\hline Alto & 15 & 21,75 & 08 & 11,60 & 58 & 84,10 \\
\hline Moderado & 45 & 65,20 & 42 & 60,90 & 10 & 14,50 \\
\hline Baixo & 09 & 13,05 & 19 & 27,50 & 01 & 1,40 \\
\hline
\end{tabular}

$\mathrm{Na}$ dimensão que analisou a $\mathrm{DE}$, foi observada maior frequência no nível moderado, registrando total de 
$60,90 \%$ ( $\mathrm{n}=42$ ), na segunda ( $\left.2^{\mathrm{a}}\right)$ colocação foi evidenciada a presença de nível baixo, constituindo $27,50 \%(\mathrm{n}=19)$, e, na última colocação, foi constatada a presença de nível alto com $11,60 \%(\mathrm{n}=08)$.

Já quando analisada a dimensão RP, foi observada a predominância de nível alto que somou $84,10 \%(\mathrm{n}=58)$, na segunda $\left(2^{\mathrm{a}}\right)$ colocação foi identificada a presença do nível moderado que somou um total de $14,50 \%(\mathrm{n}=10)$ e, na última colocação, foi observada a presença do nível baixo que registrou um total de $1,40 \%(n=01)$.

\section{Discussão}

No presente estudo, foi evidenciado que $62,30 \%$ $(n=43)$ dos profissionais de enfermagem entrevistados eram do gênero feminino. Nesse sentido, essa práxis profissional é exercida em sua maioria por pessoas do sexo feminino, característica predominante da enfermagem, conforme consagrada na literatura científica e observada em pesquisas desenvolvidas por Meneghini, Paz e Lautert (2011).

A maior frequência de pessoas que desenvolvem diagnóstico da $\mathrm{SB}$, em qualquer um de seus níveis constitutivos, consiste em profissionais de enfermagem pertencentes à faixa etária compreendida entre 20 e 40 anos. Essa faixa etária representa o grupo de profissionais de enfermagem constituintes da maior força de trabalho no campo da saúde e seus seguimentos, sendo observado um quantitativo de 76,80\% (n=53), porém, esse dado não se encontra em consonância com as pesquisas desenvolvidas por Moreira et al. (2009), ao que admite em estudo que a faixa etária de aparecimento da SB seja em faixa etária em jovens.

Foi constatado também que se trata de população jovem em fase ativa, onde 55,1\% (n=38) afirmam serem casados e a maioria possui filhos totalizando $78,6 \%$ $(\mathrm{n}=54)$, corroborando o estudo de Ritter et al. (2012).

Em nosso estudo, observou-se que 56,50\% ( $n=39)$ dos entrevistados possuíam em média de 1 a 4 anos de atividades exercidas junto à instituição participante do estudo. Paradoxalmente a esse dado, Meneghini, Paz e Lautert (2011) sustentam que, a instituição que tem em seu quadro laborativo, profissionais que possuem até uma década trabalhada em enfermagem, exercem esse ofício em instituições públicas e privadas, observando-se, na segunda, maior rotatividade dos colaboradores, uma vez que esses estão em busca de melhores condições salariais, trabalhistas e de ascensão profissional.

Dos profissionais que exercem dupla jornada de trabalho, observou-se que $17,10 \%$ declararam trabalharem em outra instituição, o que torna a responsabilidade deles muito elevada, além de obrigá-los a renunciarem vários aspectos de sua vida, tais como a convivência familiar e, também, a participação social, estando essa categoria em consonância com a literatura científica consagrada.

Os resultados evidenciaram ainda que a distribuição por níveis das dimensões proposta pela SB resultara em EE com nível moderado de 65,20\% ( $\mathrm{n}=45)$, o DE apresentou nível moderado de 60,90\% $(\mathrm{n}=42)$ e de $\mathrm{RP}$ que se demonstrou em predominância de nível alto com $84,10 \%$ ( $n=58$ ), não podendo considerar que essa amostra apresente ou sugira a presença de $\mathrm{SB}$ aos profissionais, estando essa questão em contrassenso com os estudos e análises desenvolvidos por França (2010).

Observou-se ainda que não houve presença da SB na equipe de enfermagem analisada que atua no PS, embora esta seja uma profissão considerada de muitos riscos ocupacionais, conforme defendido por Batista e Bianchi (2006). É importante ressaltar também que não foram identificados estudos sobre a SB em profissionais de enfermagem da equipe do hospital público na localidade investigada, o que dificulta a comparação entre os resultados obtidos com a literatura científica. Nesse sentido, a comparação foi realizada por meio de estudos em que os autores utilizaram MBI.

Não se pode desconsiderar que outros indivíduos apresentaram EE e DE conforme constatado junto ao processo de análise dos dados adquiridos. No grupo estudado, do universo de 69 profissionais de enfermagem da equipe do PS, observou-se que não houve a presença da SB, pois somente é indicativa quando ocorrem altas pontuações em EE, DE e baixas pontuações RP, não corroborando os estudos de Moreira et al. (2009) e França (2010).

Apesar dos resultados evidenciados não apontarem diagnóstico para a SB, e ainda, a categoria profissional em questão, possuir vários riscos ocupacionais que corroboram Meneghini, Paz e Lautert (2011), além de encontrarem em seu cotidiano com vários agentes estressantes, com a constante da tomada de decisão de inúmeros assuntos e dilemas ético-profissionais, é emergente a necessidade de realização de novos estudos e produções acadêmicas que venha a permitir melhor elucidação desse ruidoso fenômeno. 


\section{Conclusão}

A realização desta pesquisa foi significativa, sobretudo porque os objetivos foram alcançados, entretanto, não foi possível analisar a proporção de ocorrência da SB nos profissionais pesquisados, pois, para o fechamento do diagnóstico desta, seria necessário ter pontuações específicas para as três (03) dimensões. Porém, foi de grande valia o estudo, pois revelou pontos cruciais em que se deve optar pela melhora do trabalho da equipe de enfermagem.

Nesse sentido e, no decurso do processo de produção deste estudo, algumas limitações foram encontradas durante o seu desenvolvimento, a contar da disponibilidade dos profissionais em responderem aos instrumentos de coleta de dados aplicados, acrescido a essa questão da perda de entrevistados e a ausência de alguns em seu setor laborativo.

Os resultados mostraram que os profissionais entrevistados apresentaram níveis mais elevados de $\mathrm{EE}$ e DE, quando comparados à RP, na qual podemos citar pontos relevantes, de que a cidade proposta ao processamento deste estudo faz parte da capital federal e regiões administrativas (RAs), modificando dessa forma alguns resultados.

Em contrapartida com outros estudos, a questão salarial constitui ponto diferencial a outras realidades brasileiras, em virtude de os profissionais analisados mostrarem ser mais realizados profissionalmente, além da estabilidade financeira encontrada, pois o hospital em estudo se constituir de administração desenvolvida pela rede pública.

Este estudo revelou, ainda, a necessidade de realização de intervenções em relação aos aspectos, sejam eles físicos ou emocionais, pois, apesar de constatado neste estudo, os profissionais pesquisados serem realizados profissionalmente, leva-se também em consideração, que é necessária a atenção às outras dimensões relacionadas à SB.

Nesse sentido, vê-se a necessidade de reflexão em se desenvolver outros estudos e produções a respeito dessa problemática para melhor compreender os fatores que contribuem para o perfil doença/saúde dos profissionais de enfermagem, em unidades hospitalares públicas e de área regionalizada próximas da analisada neste estudo.

É considerável elucidar que a SB não constitui obstáculo somente ao sujeito, mas também circundante ao coletivo onde exerce suas atividades trabalhistas. Nes- sa perspectiva, não foi possível estabelecer comparações mais consistentes entre os resultados do estudo em questão, com os de outros trabalhos pesquisados e realizados em diversos outras unidades federativas brasileiras.

\section{Referências}

BATISTA, K. M.; BIANCHI, E. R. F. Estresse do enfermeiro em unidade de emergência. Revista Latino-Americana de Enfermagem, Ribeirão Preto, v. 14, n. 4, p. 534-539, jul./ago. 2006. doi: 10.1590/S0104-11692006000400010

FARIAS, S. M. C. et al. Caracterização dos sintomas físicos de estresse na equipe de pronto atendimento. Revista da Escola de Enfermagem da USP, São Paulo, v. 45 , n. 3 , p. $722-729$, jun. 2011. doi: 10.1590/S008062342011000300025

FRANÇA, F. M. de. Estudo sobre síndrome de Burnout em profissionais de enfermagem em dois Hospitais de médio porte no município de Cáceres -MT. 2010. 120 f. Dissertação (Mestrado) - Programa de Pós-Graduação em Ciências da Saúde, Universidade de Brasília, Brasília, 2010.

MASLACH, C.; JACKSON, S. E. The measurement of experienced burnout. Journal of Occupational Behaviour, Malden, v. 2, n. 2, p. 99-113, Apr. 1981. doi: 10.1002/ job.4030020205

MENEGHINI, F.; PAZ, A. A.; LAUTERT, L. Fatores ocupacionais associados aos componentes da síndrome de Burnout em trabalhadores de enfermagem. Revista Texto e Contexto: Enfermagem, Florianópolis, v. 20 , n. 2, p. 225-233, abr./jun. 2011. doi: 10.1590/S010407072011000200002

MOREIRA, D. S. et al. Prevalência da síndrome de Burnout em trabalhadores de enfermagem de um hospital de grande porte da Região Sul do Brasil. Cadernos de Saúde Pública, Rio de Janeiro, v. 25, n. 7, p. 1559-1568, jul. 2009. doi: 10.1590/S0102-311X2009000700014

RITTER, R. S. et al. Correlações de variáveis do Inventário de Burnout de Maslach em profissionais de emergência hospitalar. Revista Enfermería Global, Múrcia, v. 11 , n. 27 , p. 210-223, jul. 2012. doi: 10.4321/S169561412012000300012 . 
RODRIGUES, A. M. S. et al. O impacto das condições e jornada de trabalho na saúde dos trabalhadores de enfermagem. Revista de Pesquisa: Cuidado é Fundamental, Rio de Janeiro, v. 4, n. 4, p. 2867-2873, out./dez. 2012.
TAMAYO, M. R. Burnout: implicações das fontes organizacionais de desajuste indivíduo-trabalho em profissionais da enfermagem. Revista Psicologia: Reflexão e Crítica, Porto Alegre, v. 22, n. 3, p. 474-482, out. 2009. doi: 10.1590/S0102-79722009000300019 